\title{
Dense SDM (12-Core × 3-Mode) Transmission Over 527 km With 33.2-ns Mode- Dispersion Employing Low-Complexity Parallel MIMO Frequency-Domain Equalization
}

\author{
Shibahara, Kohki; Lee, Doohwan; Kobayashi, Takayuki; Mizuno, Takayuki; Takara, Hidehiko; Sano, \\ Akihide; Kawakami, Hiroto; Miyamoto, Yutaka; Ono, Hirotaka; Oguma, Manabu
}

Total number of authors:

19

Published in:

Journal of Lightwave Technology

Link to article, DOI:

10.1109/JLT.2015.2463102

Publication date:

2016

Document Version

Publisher's PDF, also known as Version of record

Link back to DTU Orbit

Citation $(A P A)$ :

Shibahara, K., Lee, D., Kobayashi, T., Mizuno, T., Takara, H., Sano, A., Kawakami, H., Miyamoto, Y., Ono, H., Oguma, M., Abe, Y., Matsui, T., Fukumoto, R., Amma, Y., Hosokawa, T., Matsuo, S., Saitoh, K., Yamada, M., \& Morioka, T. (2016). Dense SDM (12-Core × 3-Mode) Transmission Over $527 \mathrm{~km}$ With 33.2-ns Mode-Dispersion Employing Low-Complexity Parallel MIMO Frequency-Domain Equalization. Journal of Lightwave Technology, 34(1), 196-204. https://doi.org/10.1109/JLT.2015.2463102

\section{General rights}

Copyright and moral rights for the publications made accessible in the public portal are retained by the authors and/or other copyright owners and it is a condition of accessing publications that users recognise and abide by the legal requirements associated with these rights.

- Users may download and print one copy of any publication from the public portal for the purpose of private study or research.

- You may not further distribute the material or use it for any profit-making activity or commercial gain

- You may freely distribute the URL identifying the publication in the public portal 


\title{
Dense SDM (12-Core $\times 3$-Mode) Transmission Over 527 km With 33.2-ns Mode-Dispersion Employing Low-Complexity Parallel MIMO Frequency-Domain Equalization
}

\author{
Kohki Shibahara, Doohwan Lee, Takayuki Kobayashi, Member, IEEE, Takayuki Mizuno, Member, IEEE, \\ Hidehiko Takara, Member, IEEE, Akihide Sano, Member, IEEE, Hiroto Kawakami, Member, IEEE, Member, OSA, \\ Yutaka Miyamoto, Member, IEEE, Hirotaka Ono, Senior Member, IEEE, Member, OSA, Manabu Oguma, \\ Yoshiteru Abe, Takashi Matsui, Ryohei Fukumoto, Yoshimichi Amma, Tsukasa Hosokawa, \\ Shoichiro Matsuo, Member, IEEE, Member, OSA, Kunimasa Saitoh, Member, IEEE, Member, OSA, \\ Makoto Yamada, Senior Member, IEEE, and Toshio Morioka, Member, IEEE, Fellow, OSA
}

(Post-Deadline)

\begin{abstract}
We propose long-haul space-division-multiplexing (SDM) transmission systems employing parallel multiple-input multiple-output (MIMO) frequency-domain equalization (FDE) and transmission fiber with low differential mode delay (DMD). We first discuss the advantages of parallel MIMO FDE technique in long-haul SDM transmission systems in terms of the computational complexity, and then, compare the complexity required for parallel MIMO FDE as well as the conventional time-domain equalization techniques. Proposed parallel MIMO FDE that employs low baud rate multicarrier signal transmission with a receiver-side FDE enables us to compensate for 33.2-ns DMD with considerably low-computational complexity. Next, we describe in detail the newly developed fiber and devices we used in the conducted experiments. A graded-index (GI) multicore few-mode fiber (MC-
\end{abstract}

Manuscript received June 1, 2015; revised July 1, 2015; accepted July 9, 2015. Date of publication July 29, 2015; date of current version January 24, 2016. Part of this research utilized results from research commissioned by the National Institute of Information and Communications Technology of Japan

K. Shibahara, D. Lee, T. Mizuno, H. Takara, A. Sano, H. Kawakami, and Y. Miyamoto are with the NTT Network Innovation Laboratories, NTT Corporation, Yokosuka 239-0847, Japan (e-mail: shibahara.kouki@lab. ntt.co.jp; lee.doohwan@lab.ntt.co.jp; mizuno.takayuki@lab.ntt.co.jp; takara hidehiko@lab.ntt.co.jp; sano.akihide@lab.ntt.co.jp; kawakami.hiroto@lab. ntt.co.jp; miyamoto.yutaka@lab.ntt.co.jp).

T. Kobayashi and T. Matsui are with the NTT Access Network Service Systems Laboratories, NTT Corporation, Tsukuba 305-0805, Japan (e-mail: kobayashi.takayuki@lab.ntt.co.jp; matsui.takashi@lab.ntt.co.jp).

H. Ono and M. Oguma are with the NTT Device Technology Laboratories, NTT Corporation, Atsugi 243-0198, Japan (e-mail: ono.hirotaka@lab.ntt.co.jp; oguma.manabu@lab.ntt.co.jp).

Y. Abe is with the NTT Device Innovation Center, NTT Corporation, Atsugi 243-0198, Japan (e-mail: abe.yoshiteru@lab.ntt.co.jp).

R. Fukumoto, Y. Amma, T. Hosokawa, and S. Matsuo are with the Advanced Technology Laboratory, Fujikura Ltd., Chiba 285-8550, Japan (e-mail: ryohei.fukumoto@jp.fujikura.com; yoshimichi.amma@jp.fujikura com; tsukasa.hosokawa@jp.fujikura.com; shoichiro.matsuo@jp.fujikura.com).

K. Saitoh is with the Hokkaido University, Sapporo 060-0814, Japan (e-mail: ksaitoh@ist.hokudai.ac.jp).

M. Yamada is with the Osaka Prefecture University, Sakai 599-8531, Japan (e-mail:myamada@eis.osakafu-u.ac.jp).

T. Morioka is with the Technical University of Denmark, Kgs. Lyngby 2800 , Denmark (e-mail: tomo@fotonik.dtu.dk).

Color versions of one or more of the figures in this paper are available online at http://ieeexplore.iee.org.

Digital Object Identifier 10.1109/JLT.2015.2463102
FMF) suppressed the accumulation of DMD as well as intercore crosstalk. Mode dependent loss/gain effect was also mitigated by employing both a ring-core FM erbium-doped fiber amplifier and a free-space optics type gain equalizer. By combining these advanced techniques together, we finally demonstrate 12 -core $\times 3$ mode dense SDM transmission over 527-km GI MC-FMF without optical DMD management.

Index Terms-Differential mode delay (DMD), few-mode fiber (FMF), frequency-domain processing, multicore fiber (MCF), space division multiplexing.

\section{INTRODUCTION}

$\mathbf{S}$ PACE division multiplexing (SDM) techniques have contributed to the substantial enhancement of transmission capacity per optical fiber by utilizing multi-core fiber (MCF) and/or few-mode fiber (FMF) [1]-[12]. Spatial multiplicity has risen to $M_{\mathrm{MCF}}=36$ using $\mathrm{MCF}$ [10] and $M_{\mathrm{FMF}}=15$ using FMF [12], where $M_{\mathrm{MCF}}$ and $M_{\mathrm{FMF}}$ are respectively the number of cores and spatial modes. We have previously demonstrated unrepeated $40.4 \mathrm{~km}$ dense SDM (DSDM) transmission with spatial multiplicity of 36 (12-core $\times 3$-mode) by computationallyefficient parallel multiple-input multiple-output (MIMO) equalization using low baud rate multi-carriers [6].

Increasing the transmission reach for DSDM signals is essential for the practical application of DSDM systems. The most important issues for realizing such long-haul DSDM MC-FMF transmission include how to accommodate the accumulation of inter-core crosstalk for MCF transmission, and that of differential mode delay (DMD) for FMF transmission. As already demonstrated in [13] and [14], the inter-core crosstalk is effectively suppressed by employing propagation-direction interleaved transmission that alternates propagation direction between adjacent cores. To combat the DMD accumulation, most previous studies on long-haul FMF transmission have taken a DMD management approach. In [7], 900-km FMF transmission was achieved by canceling DMD with concatenated multiple opposite-signs-DMD fiber segments. In [8], it was 


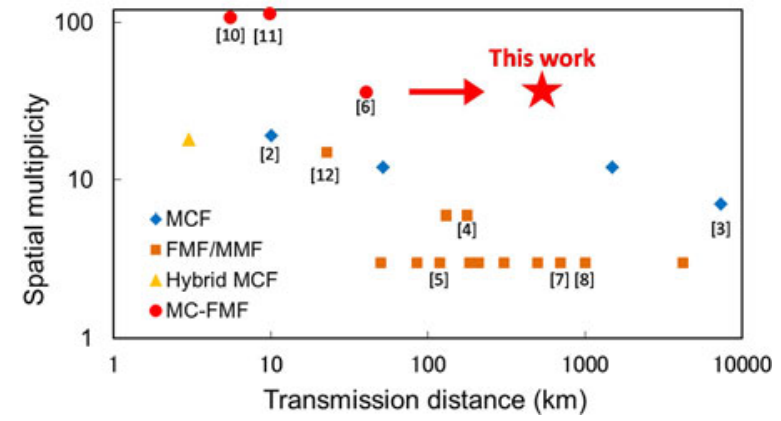

Fig. 1. SDM WDM transmission distance versus spatial multiplicity.

reported that 1000-km transmission with low-DMD FMF had been achieved without optical DMD compensation. These required high-complexity digital DMD time-domain equalization (TDE), because optical approaches perform non-perfect DMD compensation/reduction. In a weakly-coupled regime, it was demonstrated in [11] that dividing a single MIMO equalizer into "partial MIMO" equalizers in which TDE processing was carried out for lower- and higher-order mode signals independently enabled to reduce the equalizer complexity with the optical signal-to-noise ratio penalty of $1 \mathrm{~dB}$. As another approach, the use of frequency-domain equalization (FDE) techniques is promising to mitigate the equalizer complexity [9], [15], [16].

In this paper, we propose to apply a novel low-complexity DMD compensation technique using low baud rate multicarrier signal with parallel MIMO equalization. Digital compensation for DMD of over 30 ns was achieved by employing the low-complexity parallel MIMO and FDE, in combination with the newly-developed graded-index (GI) MC-FMF with low DMD, and a low mode-dependent gain FM erbium-doped fiber amplifier (EDFA). Using these technologies, we demonstrate 527-km MC-FMF DSDM inline-repeated transmission without DMD management with spatial multiplicity of 36 (12-core $\times$ 3 -mode), resulting in an achieved spectral efficiency of 2.62 $\mathrm{b} / \mathrm{s} / \mathrm{Hz} /$ core/mode. The rest of the paper is organized as follows. Section II discusses the significance of equalization for DMD in long-haul DSDM transmission, and its complexity reduction by parallel MIMO TDE and FDE. Section III provides a description of equalization algorithms for DMD and their required complexity. In Section IV, we describe the experimental setup we used, including the optical devices and signal processing technique used in the experiment. The experiment results are shown in Section V, and Section VI concludes the paper with a summary.

\section{PARALlel MIMO FDE FOR LONG-HaUl DSDM TRANSMISSION WITHOUT DMD MANAGEMENT}

We first discuss how we have developed an equalization algorithm to achieve long-haul DSDM transmission and clarify its significance in DSDM transmission. Fig. 1 shows the results achieved in recent SDM WDM transmission experiment. In this work, we successfully transmitted DSDM signals with the spatial multiplicity of 36 over $500 \mathrm{~km}$, which was more than ten times the distance relative to our previous work. We addressed the increase of DMD by implementing parallel MIMO FDE. As transmission reach increases or signal baud rate becomes

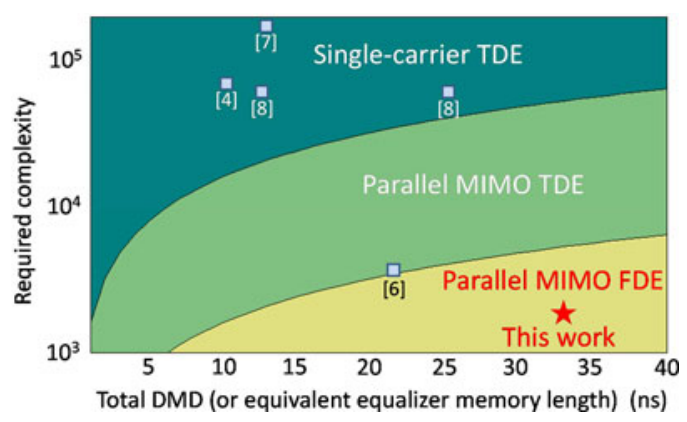

Fig. 2. Required complexity for DMD compensation in recent FMF.

higher, the required computational complexity for digital DMD compensation increases. One of the promising solutions to this is employing low baud rate multi-carrier signals with receiver-side parallel MIMO TDE [6]. However, as we will see in Section III, the complexity scales linearly with increased DMD when a TDE technique is used. Adaptive FDE algorithms for fiber-optic communication systems were proposed in [15] and [16]. They used the well-known feature of FDE; it effectively reduces the computational complexity since computation of a convolution in the time domain is replaced by FFT-based scalar multiplication [17].

We implemented FDE in conjunction with low baud rate multi-carrier signals, which we call here "parallel MIMO FDE", to further decrease the complexity of DMD compensation. Fig. 2 depicts the required computational complexity per carrier per mode which was defined by complex multiplications for calculating output/equalizer-updating and FFT/IFFT as a function of total DMD (or equivalent equalizer memory length). We divided the area in the Fig. 2 into three regions for digital DMD compensation: single-carrier (SC) TDE region, parallel MIMO TDE region [6], and parallel MIMO FDE region. The borders were set under the assumption that 10-Gbaud-SC or 10-FDM 1-Gbaud multi-carrier signals are transmitted through FMF with DMD. Fig. 2 is helpful to approximately estimate the required complexity of each equalization scheme for signals with arbitrary symbol rate. Whereas the total DMD of $33.2 \mathrm{~ns}$ in our experiments was the largest among recent SDM experiments, the use of parallel MIMO FDE significantly suppressed the complexity and enabled us to achieve transmission with considerably lowercomplexity than was achieved with other methods. Switching SC-TDE to parallel MIMO FDE is expected to reduce the complexity by a factor of around 33 .

\section{ADAPTIVE AlgORITHMS FOR DMD EQUALIZATION}

In this section, we briefly describe three adaptive equalization algorithms: SC-TDE, parallel MIMO TDE [6], and parallel MIMO FDE. We also derive and compare the required computational complexity for three schemes. The complexity is defined as the number of complex multiplications per symbol per mode in each scheme in this section [15], [16].

\section{A. Single-Carrier TDE}

We start with the definition of $\Delta \tau, N_{t}, N_{m}, R_{o}$, and $R_{s}$ which respectively denote total DMD, the number of equalizer taps for 
SC-TDE, the number of spatial and polarization modes, the oversampling rate, and the symbol rate. Note that only integer value for $R_{o}$ is considered in this paper. If positive and negative DMD effects are taken into account, $N_{t}$ is equal to $2 \Delta \tau R_{o} R_{s}$ If the $k$ th received signal for $i$ th mode is denoted as $y_{i, k}$, the $k$ th received signal vector for the $i$ th mode is expressed as $\boldsymbol{y}_{i, k}=\left[y_{i, k-\left\lfloor N_{t} / 2\right\rfloor} y_{i, k-\left\lfloor N_{t} / 2\right\rfloor+1} \ldots y_{i, k+\left\lceil N_{t} / 2\right\rceil-1}\right]^{\mathrm{T}}$, where $\mathrm{T},\lfloor\cdot\rfloor$, and $[\cdot]$ respectively denote the transpose operation, the floor function, and the ceiling function. The complex-valued $N_{t} N_{m} \times N_{m}$ equalizer matrix $\mathrm{W}$ is defined as

$$
\mathrm{W}=\left[\boldsymbol{w}_{i j}\right]
$$

where $\boldsymbol{w}_{i j}$ denotes the $(i, j)$-th column vector of $\mathbf{W}$. The $k$ th symbol of the equalizer output for the $i$ th mode $x_{i, k}$ is obtained as

$$
x_{i, k}=\sum_{j=1}^{N_{m}} \boldsymbol{w}_{i j}{ }^{\mathrm{T}} \boldsymbol{y}_{j, k} .
$$

Note that $\boldsymbol{y}_{i, k}$ and $\boldsymbol{w}_{i j}$ are vectors of length $N_{t}$. We employ a data-aided or decision-directed least mean square (LMS) method for equalizer update. Thus, using the $k$ th desired output symbol for $i$ th mode $d_{i, k}$, the error signal $e_{i, k}$ becomes

$$
e_{i, k}=d_{i, k}-x_{i, k} \text {. }
$$

The corresponding equalizer updating equation with the stepsize parameter $\mu$ is derived as

$$
\boldsymbol{w}_{i j} \leftarrow \boldsymbol{w}_{i j}+\mu e_{i, k} \boldsymbol{y}_{j, k}{ }^{*}
$$

From (1)-(4), we find that $N_{t} N_{m}$ multiplications are needed for one symbol output per mode and $\left(N_{t} N_{m}+1\right)$ multiplications are needed for equalizer updating. Thus the complexity for SCTDE is calculated as

$$
\begin{aligned}
C_{\mathrm{SC}-\mathrm{TDE}} & =2 N_{t} N_{m}+1 \\
& =4 \Delta \tau R_{o} R_{s} N_{m}+1 .
\end{aligned}
$$

\section{B. Parallel MIMO TDE}

If we divide an optical carrier into $P$ subcarriers, each subcarrier has to be driven at a symbol rate of $R_{s} / P$ to keep the data rate unchanged. Thus the equalizer length for multicarrier equalization algorithm $N_{t}^{\prime}$ becomes

$$
N_{t}^{\prime}=\frac{N_{t}}{P}=\frac{2 \Delta \tau R_{o} R_{s}}{P} .
$$

The adaptation algorithm for the parallel MIMO TDE is basically identical to that for SC-TDE.

While the number of symbols contained in a unit time for one subcarrier is decreased by a factor of $P$ due to the lower symbol rate, the number of subcarriers increases by a factor of $P$. Consequently, the complexity for parallel MIMO TDE is calculated as

$$
\begin{aligned}
C_{\mathrm{P}-\mathrm{TDE}} & =\left(2 N_{t}^{\prime} N_{m}+1\right)\left(\frac{1}{P}\right) P \\
& =\frac{4 \Delta \tau R_{o} R_{s} N_{m}}{P}+1 .
\end{aligned}
$$

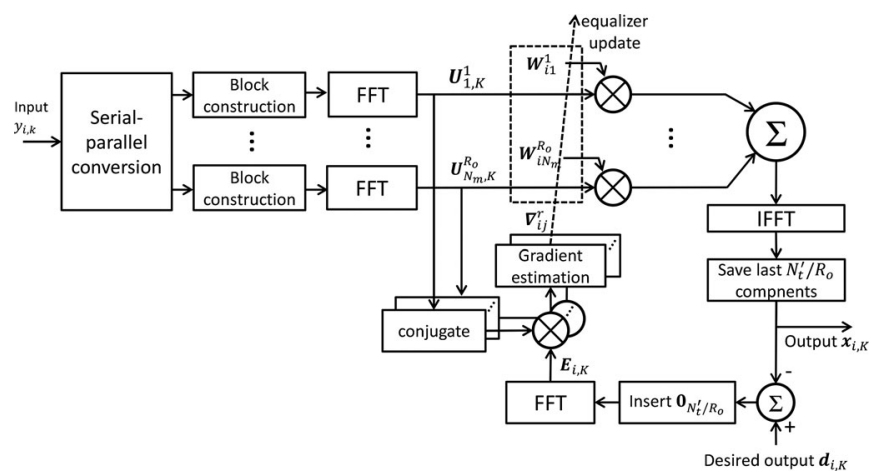

Fig. 3. The schematic processing flow of our FDE scheme.

\section{Parallel MIMO FDE}

We here consider parallel MIMO FDE that applies the overlap-and-save method with a $50 \%$ overlap ratio for simplicity [18]. The schematic processing flow of our FDE scheme is illustrated in Fig. 3. Note that an equalizer is split into $R_{o}$ sub-equalizers $\boldsymbol{w}_{i j}^{r}$ with equalizer length of $N_{t}^{\prime} / R_{o}$ where superscript $r$ represents an oversampling index $\left(1 \leq r \leq R_{o}\right)$, and that the block processing with the block size of $2 N_{t}^{\prime} / R_{o}$ outputs the $N_{t}^{\prime} / R_{o}$ symbols. The first step is the converting the received serial sequence for each mode into $R_{o}$ parallel sequences. Then the $K$ th block input $\boldsymbol{U}_{i, K}^{r}$ is constructed by using $N_{t}^{\prime} / R_{o}$ samples from the current block and $N_{t}^{\prime} / R_{o}$ samples from the previous block.

$$
\boldsymbol{U}_{i, K}^{r}=\operatorname{diag}\left[F\left(y_{i, K N_{t}^{\prime}-N_{t}^{\prime}}^{r} y_{i, K N_{t}^{\prime}-N_{t}^{\prime}+1}^{r} \cdots y_{i, K N_{t}^{\prime}+N_{t}^{\prime}-1}^{r}\right)\right]
$$

where $\operatorname{diag}(\cdot)$ makes a diagonal matrix with a vector input and $F(\cdot)$ denotes FFT operation. A sub-equalizer in the frequency domain $\boldsymbol{W}_{i j}^{r}$ is derived from the corresponding time-domain quantities as

$$
\boldsymbol{W}_{i j}^{r}=F\left[\boldsymbol{w}_{i j}^{r} \mathbf{0}_{N_{t}^{\prime} / R_{o}}\right]
$$

where $\mathbf{0}_{N_{t}^{\prime} / R_{o}}$ is a column vector with $N_{t}^{\prime} / R_{o}$-length zeros. The $K$ th block equalizer output for the $i$ th mode $\boldsymbol{x}_{i, K}$ is obtained as

$$
\boldsymbol{x}_{i, K}=\text { last } N_{t}^{\prime} / R_{o} \text { components of } F^{-1}\left[\sum_{r=1}^{R_{o}} \sum_{j=1}^{N_{m}} \boldsymbol{U}_{j, K}^{r} \boldsymbol{W}_{i j}^{r}\right]
$$

where $F^{-1}(\cdot)$ denotes IFFT operation. Note that $\boldsymbol{U}_{j, K}^{r}$ and $\boldsymbol{W}_{i j}^{r}$ have respectively size of $2 N_{t}^{\prime} / R_{o} \times 2 N_{t}^{\prime} / R_{o}$ and $2 N_{t}^{\prime} / R_{o} \times 1$. By expanding (3) to the vector form, the error signal vector for the $K$ th block $\boldsymbol{E}_{i, K}$ is calculated in the frequency domain as

$$
\boldsymbol{E}_{i, K}=F\left[\mathbf{0}_{N_{t}^{\prime} / R_{o}}\left(\boldsymbol{d}_{i, K}-\boldsymbol{x}_{i, K}\right)\right]
$$

where $\boldsymbol{d}_{i, K}$ and $\boldsymbol{x}_{i, K}$ are respectively the $K$ th desired output vector and the $K$ th output vector for $i$ th mode. The block gradient estimate $\nabla_{i j}^{r}$ becomes

$$
\boldsymbol{\nabla}_{i j}^{r}=\text { first } N_{t}^{\prime} / R_{o} \text { components of } F^{-1}\left[\boldsymbol{U}_{j, K}^{r}{ }^{\mathrm{H}} \boldsymbol{E}_{i, K}\right]
$$




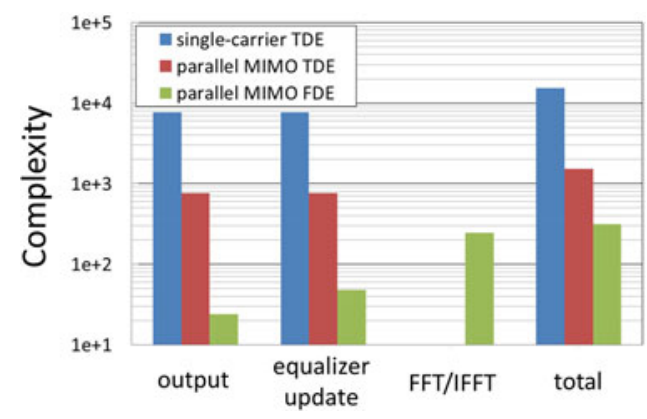

Fig. 4. Comparison of the required complexity in each step for three equalization schemes.

where $\mathrm{H}$ denotes the complex conjugate transpose operation. Thus we finally get the equalizer updating equation as

$$
\boldsymbol{W}_{i j}^{r} \leftarrow \boldsymbol{W}_{i j}^{r}+\mu F\left[\boldsymbol{\nabla}_{i j}^{r} \mathbf{0}_{N_{t}^{\prime} / R_{o}}\right]
$$

Equations (8)-(13) indicate that we need $2 N_{t}^{\prime} N_{m}$ multiplications for output calculation and $4 N_{t}^{\prime} N_{m}$ for equalizer updating. The total number of FFT/IFFT operations becomes $\left(2+R_{o}+2 R_{o} N_{m}\right)$ including $R_{o}$ FFT in the input sequence transform, 2 FFT/IFFT in the processing to derive $\boldsymbol{E}_{i, K}$, and $2 R_{o} N_{m}$ FFT/IFFT in the gradient estimation and the equalizer updating. Assuming the implementation of FFT/IFFT with FFT-size of $L$ by the radix-2 algorithm that needs $(L / 2) \log _{2} L$ complex multiplications, and recalling that FFT-size is equal to $2 N_{t}^{\prime} / R_{o}$, we can calculate the complexity for parallel MIMO FDE as

$$
\begin{aligned}
C_{\mathrm{P}-\mathrm{FDE}} & =\left(2+R_{o}+2 R_{o} N_{m}\right) \log _{2}\left(\frac{2 N_{t}^{\prime}}{R_{o}}\right)+6 N_{m} R_{o} \\
& =\left(2+R_{o}+2 R_{o} N_{m}\right) \log _{2}\left(\frac{4 \Delta \tau R_{s}}{P}\right)+6 N_{m} R_{o} .
\end{aligned}
$$

Fig. 4 compares the required complexity in each step for adaptive equalization schemes. In the figure, we respectively set $N_{t}, N_{m}, R_{o}$ and $P$ to $1280,6,2$, and 10 . The figure shows that the complexity in the parallel MIMO FDE scheme is reduced mainly due to simplification of the output and equalizerupdating calculations. We also found that a computationallyeffective FFT/IFFT algorithm would contribute to further reduction of the complexity [19], although we do not discuss this here since it is out of the scope of this work.

\section{EXPERIMENTAL SETUP}

Next, we conducted a DSDM transmission experiment. The experimental setup is depicted in Fig. 5(a). At the transmitter, a test and 19 dummy channels were respectively generated by a tunable external-cavity laser with a $25-\mathrm{kHz}$ linewidth and by DFB lasers with a 2-MHz linewidth. The 12.5- GHz-spaced CW carriers (1556.0-1557.9 nm) were separately multiplexed into even/odd channels. The 1.04-GHz-spaced 10-FDM multicarrier QPSK signals were digitally generated, each of them was driven at 1-Gbaud and reshaped by a root-raised-cosine filter with a roll-off factor of 0.01. Each mode signal was modulated independently by different binary patterns using pieces of the PRBS of length $2^{23}-1$. The frame of 31250 symbol-length comprised $1.63 \%$ overhead for the training sequence, $20 \%$ overhead for forward error correction (FEC), and a payload. The transmitter frequency response was pre-equalized in a zero-forcing manner. The even/odd channels were combined by $12.5 / 25 \mathrm{GHz}$ interleave filters and then fed into a PDM-emulator with $275 \mathrm{~ns}$ delay to create the PDM channels. This yielded 20-ch 12.5GHz-spaced $40 \mathrm{~Gb} / \mathrm{s}$ PDM-QPSK signals, resulting in a net data rate of $32.79 \mathrm{~Gb} / \mathrm{s}$ and $\mathrm{SE}$ of $2.62 \mathrm{~b} / \mathrm{s} / \mathrm{Hz} /$ core/mode. An optical spectrum with $20 \mathrm{MHz}$ resolution is shown in Fig. 5(b). The independently-modulated PDM signals were each split into three ports, delayed, preamplified, and input to $\mathrm{LP}_{01}, \mathrm{LP}_{11 a}$, and $\mathrm{LP}_{11 b}$ input ports of three silica planar lightwave circuit (PLC) mode multiplexers (MUXs). The mode-multiplexed signals were each split by a $1 \times 4 \mathrm{FM}$ splitter, delayed, and input to 12 recirculating loops operated synchronously. All 36 DSDM tributaries at the fan-in (FI) input of the MC-FMF had their power set at $-9 \mathrm{dBm} /$ wavelength/core/mode.

The transmission line consisted of a newly developed GI low-DMD MC-FMF with 52.7-km length. The DMD was varied core-to-core in the range from -41 to $63 \mathrm{ps} / \mathrm{km}$ for the C-band where positive (negative) DMD corresponds to the case in which $\mathrm{LP}_{01}\left(\mathrm{LP}_{11}\right)$ mode signals propagate earlier. The physical parameters of the fiber are summarized in Table I. The FM cores were designed with two types of trench-assisted GI profiles having different propagation constants placed next to each other in a square lattice arrangement with a view to minimizing core-to-core crosstalk (see Fig. 5(c)). The core pitch, the cladding diameter, and the worst core-to-core crosstalk after 500-km transmission were $43,230 \mu \mathrm{m}$, and $-48.4 \mathrm{~dB}$ between $\mathrm{LP}_{11}$ modes, respectively, and the dispersion of the $\mathrm{LP}_{01}$ mode was $19.8 \mathrm{ps} / \mathrm{nm} / \mathrm{km}$ at $1550 \mathrm{~nm}$. The attenuation loss at 1550 $\mathrm{nm}$ was 0.218 and $0.228 \mathrm{~dB} / \mathrm{km}$ for the $\mathrm{LP}_{01}$ and $\mathrm{LP}_{11}$ modes, respectively, and the effective area at $1550 \mathrm{~nm}$ was $110 \mu \mathrm{m}^{2}$ for the $\mathrm{LP}_{01}$ mode. The total span loss at $1550 \mathrm{~nm}$ of the transmission fiber with physical contact type fan-in/fan-out (FI/FO) devices was 12.0-13.4 $\mathrm{dB}$ for the $\mathrm{LP}_{01}$ mode and 11.9-14.9 dB for the $\mathrm{LP}_{11}$ mode. Each loop included a ring-core FM-EDFA and a free-space optics type mode dependent loss (MDL) equalizer. A FM-EDFA has a gain of $>18 \mathrm{~dB}$, a typical differential modal gain of $<1.4 \mathrm{~dB}$, and the noise figure of $<5.2 \mathrm{~dB}$ for $\mathrm{LP}_{01}$ mode and of $<5.8 \mathrm{~dB}$ for $\mathrm{LP}_{11}$ mode [20]. The total input and output power of a FM-EDFA were -4.4 and $16.7 \mathrm{dBm} /$ core on average, respectively. A MDL equalizer that compensates for the loss difference of $3 \mathrm{~dB}$ between $\mathrm{LP}_{01}$ and $\mathrm{LP}_{11}$ modes within a loop consists of a collimator pair and a small dot shaped neutral density (ND) filter. The $\mathrm{LP}_{01}$ mode signals are attenuated heavily, because its modal intensity profile is strongly overlapped with the ND filter. On the other hand, the $\mathrm{LP}_{11}$ mode signal's loss becomes small due to its small overlap-integral. Note that we fabricated and employed twelve parallel FM-EDFAs in the transmission experiment. These optical devices allowed us to suppress MDL to as low as $0.2 \mathrm{~dB}$ per loop on average.

At the receiver, the core under test was selected for each measurement through spatial demultiplexing by the FO device and then mode-demultiplexed by the PLC mode DEMUX. The 


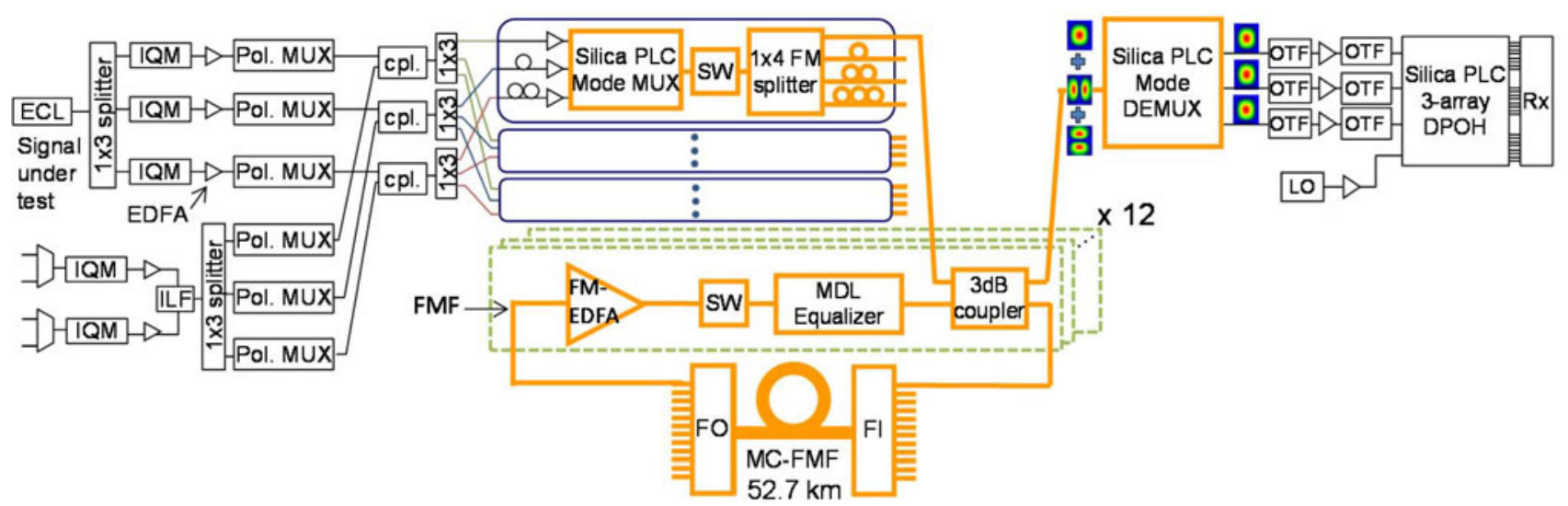

(a)

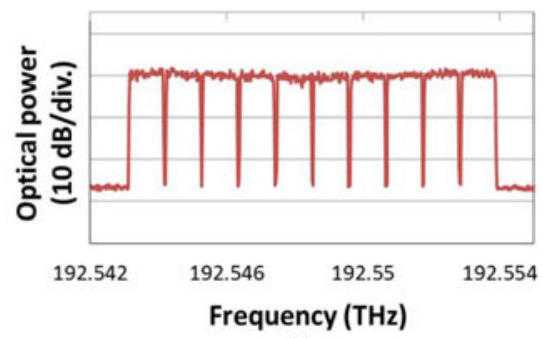

(b)

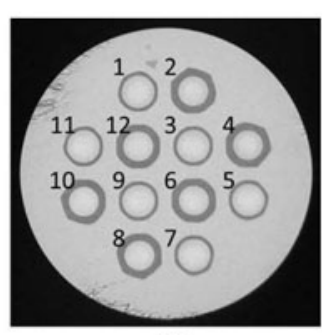

(c)

Fig. 5. (a) Experimental setup, (b) low baud rate multi-carrier signal, and (c) cross section of 12 -core $\times 3$-mode low-DMD MC-FMF transmission fiber.

TABLE I

CHARACTERISTICS OF THE MC-FMF

\begin{tabular}{lcc}
\hline \hline Parameters & Spatial mode & Value \\
\hline Attenuation & $\mathrm{LP}_{01}$ & $0.218 \mathrm{~dB} / \mathrm{km}$ \\
& $\mathrm{LP}_{11}$ & $0.228 \mathrm{~dB} / \mathrm{km}$ \\
Effective area & $\mathrm{LP}_{01}$ & $110 \mu \mathrm{m}^{2}$ \\
Inter-core crosstalk with FI/FO devices & $\mathrm{LP}_{11}$ & $<-48.4 \mathrm{~dB}$ \\
DMD in the C-band & Maximum & $63 \mathrm{ps} / \mathrm{km}$ \\
& Minimum & $-41 \mathrm{ps} / \mathrm{km}$ \\
Length & Average & $29 \mathrm{ps} / \mathrm{km}$ \\
Cladding diameter & - & $52.7 \mathrm{~km}$ \\
Core pitch & - & $230 \mu \mathrm{m}$ \\
\hline \hline
\end{tabular}

${ }^{\mathrm{a}}$ After 500-km transmission.

signals were injected to the optical tunable filters one by one for wavelength demultiplexing and input together to a PLC 3-array integrated dual polarization optical hybrid module designed for $6 \times 6$ MIMO signal processing. The received signals were digitized at $40 \mathrm{GS} / \mathrm{s}$ using a 12-ch digital storage oscilloscope, and stored in sets of $8 \mathrm{M}$ samples. Fig. 6 explains the offline parallel MIMO processing flow we employed in our experiments. After frontend error correction and chromatic dispersion compensation, out-of-band noise was removed by the first low-pass digital filter. The combined processing of frequency shift and second low-pass filtering worked as a band-pass filter to extract the target subcarrier. Then equalization and DMD compensation were carried out in a parallel processing for 10-FDM multi-carriers by using adaptive $6 \times 6$-MIMO FDE with half-symbol-spaced

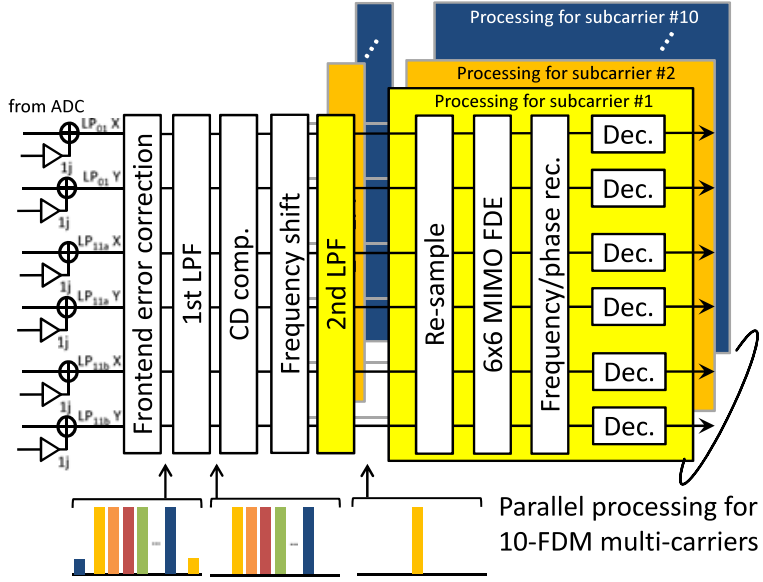

Fig. 6. Offline parallel MIMO processing flow.

taps and frequency/phase recovery. In the $6 \times 6-\mathrm{MIMO}$ FDE process, fast convergence was achieved by using a data-aided normalized-LMS-based equalizer update. The adaptation algorithm was then switched to decision-directed mode. Note that no cyclic prefix was added in our FDE scheme since we used the overlap-and-save method [18], and that the equalizer tap length for FDE was varied depending on the transmission distance to appropriately compensate DMD (e.g., 128 taps were used in $527 \mathrm{~km}$ transmission for the total DMD of $33.2 \mathrm{~ns}$ ). We used 2.5 $\mathrm{M}$ bits to count bit-error ratio (BER) per carrier per mode by means of differential decoding. Finally, the Q-factor was calculated from the measured BER. 


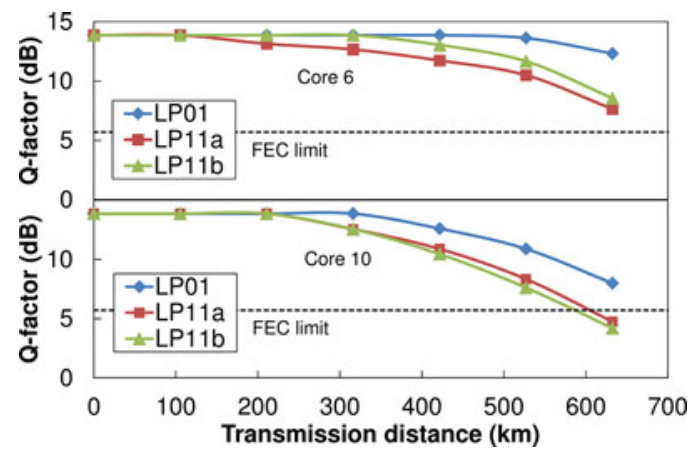

Fig. 7. Q-factor transition as a function of transmission distance for core \#6 (top panel) and \#10 (bottom panel).

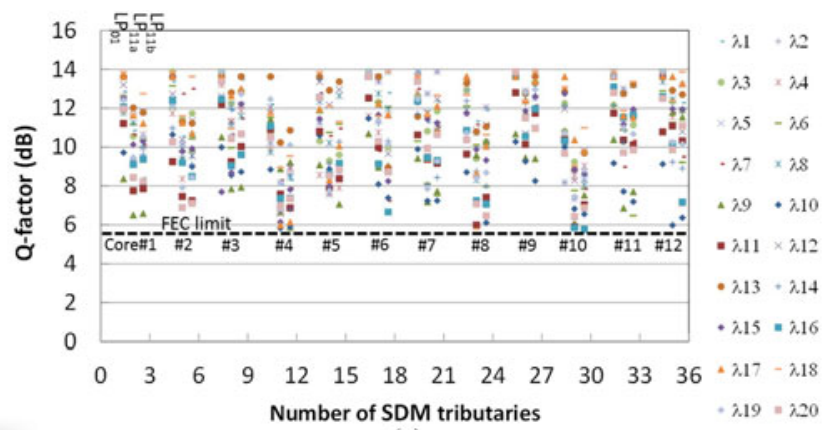

(a)

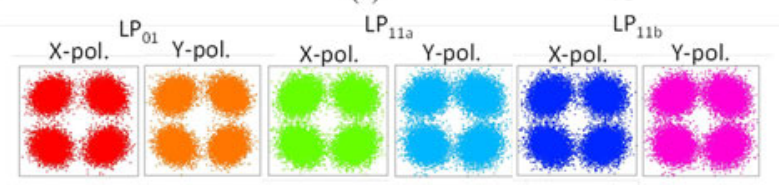

(b)

Fig. 8. (a) Q-factors after $527-\mathrm{km}$ transmission. The number of SDM tributaries is defined as $(n-1) \times 3+m$, where $n$ and $m$ is the core number and the mode number respectively ( $m=1$ for $\mathrm{LP}_{01}, m=2$ for $\mathrm{LP}_{11 a}$, and $m=3$ for $\mathrm{LP}_{11 b}$ ), (b) typical constellations for all spatial and polarization modes.

\section{TRANSMISSION RESULTS}

We examined the transmission characteristics of core \#6 and $\# 10$, which, respectively, have the largest and average DMD among all cores. Fig. 7 shows the Q-factors as a function of transmission distance for core \#6 and \#10 of $\lambda 11$ of the WDM channels. The achievable transmission distance of core \#10 was shorter than that of core \#6 which, as mentioned above, has the largest DMD. The residual MDL of the core \#10 recirculating loop was $0.35 \mathrm{~dB} /$ loop, whereas the average for all the cores was $0.2 \mathrm{~dB} /$ loop. This larger MDL could be the main factor that limited the transmission distance of core \#10. Fig. 8(a) shows the measured Q-factor performance for all channels after 527$\mathrm{km}$ transmission. We confirmed that the measured Q-factors for all 36 SDM tributaries for the 20 wavelengths exceeded the Qlimit of $5.7 \mathrm{~dB}$ of LDPC convolutional codes using a layered decoding algorithm with 20\% FEC overhead [21]. Fig. 8(b) shows the constellations of core \#11, $\lambda 10$, and subcarrier \#4.

\section{CONCLUSION}

We have successfully achieved the 12 -core $\times 3$-mode DSDM 527-km transmission with 33.2-ns DMD. In order to compen- sate for the large DMD without optical DMD management, we implemented low complexity parallel MIMO FDE into the low baud rate multi-carrier transmission. We also employed the GI MC-FMF with DMD of $63 \mathrm{ps} / \mathrm{km}$ we had newly developed. It was found that the use of an FM-EDFA with improved differential modal gain and a mode dependent loss equalizer reduced mode-dependent gain/loss to as low as $0.2 \mathrm{~dB}$ on average. Experiment results showed that the combination of parallel MIMO FDE and GI MC-FMF is a promising solution for achieving long-distance DSDM transmission.

\section{REFERENCES}

[1] T. Morioka, "New generation optical infrastructure technologies: 'EXAT initiative' towards 2020 and beyond," presented at the OptoElectronics Communications Conf., Hong Kong, 2009, Paper FT4.

[2] J. Sakaguchi, B. J. Puttnam, W. Klaus, Y. Awaji, N. Wada, A. Kanno, T. Kawanishi, K. Imamura, H. Inaba, K. Mukasa, R. Sugizaki, T. Kobayashi, and M. Watanabe, "19-core fiber transmission of $19 \times$ $100 \times 172-\mathrm{Gb} / \mathrm{s}$ SDM-WDM-PDM-QPSK signals at 305Tb/s," presented at the Nat. Fiber Optic Engineers Conf. Los Angeles, CA, USA, Mar. 2012, Paper PDP5C-1.

[3] K. Igarashi, T. Tsuritani, I. Morita, Y. Tsuchida, K. Maeda, M. Tadakuma, T. Saito, K. Watanabe, K. Imamura, R. Sugizaki, and Suzuki, M, "1.03Exabit/s km super-Nyquist-WDM transmission over 7,326-km sevencore fiber," presented at the Eur. Conf. Exhib. Optical Communication, London, U.K., 2013, Paper PD3.E.3.

[4] R. Ryf, S. Randel, N. K. Fontaine, M. Montoliu, E. Burrows, S. Corteselli, S. Chandrasekhar, A. H. Gnauck, C. Xie, R. J. Essiambre, P. J. Winzer, R. Delbue, P. Pupalaikis, A. Sureka, Y. Sun, L. Grüner-Nielsen, R. V. Jensen, and R. Lingle, "32-bit/s/Hz spectral efficiency WDM transmission over 177-km few-mode fiber," presented at the Optical Fiber Communication Conf., Anaheim, CA, USA, 2013, Paper PDP5A-1.

[5] V. A. J. M. Sleiffer, Y. Jung, V. Veljanovski, R. G. H. van Uden, M. Kuschnerov, Q. Kang, L. Grüner-Nielsen, Y. Sun, D. J. Richardson, S. Alam, F. Poletti, J. K. Sahu, A. Dhar, H. Chen, B. Inan, A. M. J. Koonen, B. Corbett, R. Winfield, A. D. Ellis, and de H. Waardt, "73.7 Tb/s $(96 \times 3 \times 256-\mathrm{Gb} / \mathrm{s})$ mode-division-multiplexed DP-16QAM transmission with inline MM-EDFA," presented at the Eur. Conf. Exhib. Optical Communication, Amsterdam, The Netherlands, 2012, Paper Th.3.C.4.

[6] T. Mizuno, T. Kobayashi, H. Takara, A. Sano, H. Kawakami, T. Nakagawa, Y. Miyamoto, Y. Abe, T. Goh, M. Oguma, T. Sakamoto, Y. Sasaki, I. Ishida, K. Takenaga, S. Matsuo, K. Saitoh, and T. Morioka, "12-core $\times 3$-mode dense space division multiplexed transmission over $40 \mathrm{~km}$ employing multi-carrier signals with parallel MIMO equalization," presented at the Optical Fiber Communication Conf., San Francisco, CA, USA, 2014, Paper Th5B-2.

[7] R. Ryf, N. K. Fontaine, M. Montoliu, S. Randel, B. Ercan, H. Chen, S. Chandrasekhar, A. H. Gnauck, S. G. Leon-Saval, J. Bland-Hawthorn, J. R. Salazar-Gil, Y. Sun, and R. Lingle, Jr., "Photonic-lantern-based mode multiplexers for few-mode-fiber transmission," presented at the Optical Fiber Communication Conf., San Francisco, CA, USA, 2014, Paper W4J2.

[8] E. Ip, M. J. Huang, K. Bennett, Y. K. Huang, A. Tanaka, A. Korolev, K. Koreshkov, W. Wood, E. Mateo, J. Hu, and Y. Yano, " $146 \lambda \times 6 \times$ 19-Gbaud wavelength-and mode-division multiplexed transmission over $10 \times 50-\mathrm{km}$ spans of few-mode fiber with a gain-equalized few-mode EDFA," presented at the Optical Fiber Communication Conf., Anaheim, CA, USA, 2013, Paper PDP5A-2.

[9] R. Ryf, N. K. Fontaine, B. Guan, B. Huang, M. Esmaeelpour, S. Randel, A. H. Gnauck, S. Chandrasekhar, A. Adamiecki, G. Raybon, R. W. Tkach, R. Shubochkin, Y. Sun, and R. Lingle, "305-km combined wavelength and mode-multiplexed transmission over conventional graded-index multimode fibre," presented at the Eur. Conf. Exhib. Optical Communication, Cannes, France, 2014, Paper PD3.5.

[10] J. Sakaguchi, W. Klaus, J.-M. D. Mendinueta, B. J. Puttnam, R. S. Luis, Y. Awaji, N. Wada, T. Hayashi, T. Nakanishi, T. Watanabe, Y. Kokubun, T. Takahata, and T. Kobayashi, "Realizing a 36-core, 3-mode fiber with 108 spatial channels," presented at the Optical Fiber Communication Conf., Los Angeles, CA, USA, 2015, Paper Th5C-2.

[11] K. Igarashi, D. Souma, Y. Wakayama, K. Takeshima, Y. Kawaguchi, T. Tsuritani, I. Morita, and M. Suzuki, "114 space-division-multiplexed 
transmission over 9.8-km weakly-coupled-6-mode uncoupled-19-core fibers," presented at the Optical Fiber Communication Conf., Los Angeles, CA, USA, 2015, Paper Th5C-4.

[12] N. K. Fontaine, R. Ryf, H. Chen, A. V. Benitez, J. E. A. Lopez, R. A. Correa, B. Guan, B. Ercan, R. P. Scott, S. J. B Yoo, L. GrünerNielsen, Y. Sun, and R. J. Lingle, "30 × 30 mimo transmission over 15 spatial modes," presented at the Optical Fiber Communication Conf., Los Angeles, CA, USA, 2015, Paper Th5C-1.

[13] A. Sano, H. Takara, T. Kobayashi, H. Kawakami, H. Kishikawa, T. Nakagawa, Y. Miyamoto, Y. Abe, H. Ono, K. Shikama, M. Nagatani, T. Mori, Y. Sasaki, I. Ishida, K. Takenaga, S. Matsuo, K. Saitoh, M. Koshiba, M. Yamdada, H. Masuda, and T. Morioka, "409-Tb/s + 409$\mathrm{Tb} / \mathrm{s}$ crosstalk suppressed bidirectional MCF transmission over $450 \mathrm{~km}$ using propagation-direction interleaving," Opt. Express, vol. 21, no. 14, pp. 16777-16783, Apr. 2013.

[14] T. Kobayashi, H. Takara, A. Sano, T. Mizuno, H. Kawakami, Y. Miyamoto, K. Hiraga, Y. Abe, H. Ono, M. Wada, Y. Sasaki, I. Ishida, K. Takenaga, S. Matsuo, K. Saitoh, M. Yamada, H. Masuda, and T. Morioka, " $2 \times 344$ $\mathrm{Tb} / \mathrm{s}$ propagation-direction interleaved transmission over 1500-km MCF enhanced by multicarrier full electric-field digital back-propagation," presented at the Eur. Conf. Exhib. Optical Communication, London, U.K., 2013, Paper PD3.E.4.

[15] M.S. Faruk and K. Kikuchi, "Adaptive frequency-domain equalization in digital coherent optical receivers," Opt. Express, vol. 19, no. 13, pp. 12789-12798, Jun. 2011.

[16] N. Bai and G. Li, "Adaptive frequency-domain equalization for modedivision multiplexed transmission," IEEE Photon. Technol. Lett., vol. 24, no. 21, pp. 1918-1921, Sep. 2012.

[17] K. Ishihara, R. Kudo, T. Kobayashi, A. Sano, Y. Takatori, T. Nakagawa, and Y. Miyamoto, "Frequency-domain equalization for coherent optical transmission systems," presented at the Optical Fiber Communication Conf., Los Angeles, CA, USA, 2011, Paper OWW4.

[18] J. J. Shynk, "Frequency-domain and multirate adaptive filtering," IEEE Signal Process. Mag., vol. 9, no. 1, pp. 14-37, Jan. 1992.

[19] P. Duhamel, "Implementation of 'split-radix' FFT algorithms for complex, real, and real-symmetric data," IEEE Trans. Acoust. Speech Signal Process., vol. 34, no. 2, pp. 285-295, Apr. 1986.

[20] H. Ono, T. Hosokawa, K. Ichii, S. Matsuo, and Yamada,"Improvement of differential modal gain in few-mode fibre amplifier by employing ringcore erbium-doped fibre," Electron. Lett., vol. 51, no. 2, pp. 172-173, Jan. 2015.

[21] D. Chang, F. Yu, Z. Xiao, N. Stojanovic, F. N. Hauske, Y. Cai, C. Xie, L. Li, $\mathrm{X}$. Xu, and Q. Xiong, "LDPC convolutional codes using layered decoding algorithm for high speed coherent optical transmission," presented at the Optical Fiber Communication Conf., Los Angeles, CA, USA, 2012, Paper OW1H.4.

Kohki Shibahara received the B.S. degree in physics and M.S. degree in geophysics from Kyoto University, Kyoto, Japan, in 2008 and 2010, respectively.

Since 2010, he has been with the NTT Network Innovation Laboratories, Yokosuka, Kanagawa, Japan. His current research interests include spatial division multiplexing transmission systems and advanced MIMO signal processing. $\mathrm{He}$ is a Member of the Institute of Electronics, Information and Communication Engineers of Japan.

Doohwan Lee received the B.S. and M.S. degrees in electrical engineering from Seoul National University, Seoul, Korea, in 2004 and 2006, respectively, and the Ph.D. degree in electrical engineering and information systems from the University of Tokyo, Tokyo, Japan, in 2009.

From 2009 to 2012, he was with the NTT Network Innovation Laboratories, Yokosuka, Japan, as a Postdoctoral Researcher and Researcher. From 2012 to 2014, he was with the Research Center for Advanced Science and Technology, University of Tokyo as an Assistant Professor. Since 2014, he has been with the NTT Network Innovation Laboratories as a Researcher Engineer. His research interests include compressed sensing, flexible wireless systems, software-defined radio, cognitive radio, and signal processing. He received the Best Paper Award and the Best Technical Exhibition Award from the IEICE Technical Committee on Software Radio in 2011, the IEICE Communication Society Excellent Paper Award in 2012, and the Best Technical Exhibition award from SmartCom in 2014.
Takayuki Kobayashi (S'05-M'06) received the B.E. and M.E. degrees in communications engineering from Waseda University, Tokyo, Japan, in 2004 and 2006, respectively.

Since April 2006, he has been with the NTT Network Innovation Laboratories, Yokosuka, Japan. His current research interests include modulation formats, coherent detection, and high-speed fiber-optic communications systems. He is a Member of the Institute of Electronics, Information and Communication Engineers of Japan and the IEEE Photonics Society.

Takayuki Mizuno (M'04) received the B.E. degree in applied physics, the M.E. degree in crystalline materials science, and the Dr. Eng. degree in quantum engineering from Nagoya University, Nagoya, Japan, in 1998, 2000, and 2007, respectively.

In 2000, he joined the NTT Photonics Laboratories, NTT Corporation, Japan, where he was involved in the research and development of silica planar lightwave circuit optical waveguide devices, including arrayed-waveguide gratings, Mach-Zehnder interferometer-based filters and switches, and digital coherent demodulators for advanced modulation formats. He is currently a Senior Research Engineer at the NTT Network Innovation Laboratories, NTT Corporation, Yokosuka, Kanagawa, Japan. He is the Author and/or Coauthor of more than 80 journals and international conference papers, and holds more than 30 granted patents. He is a Member of the Institute of Electronics, Information and Communication Engineers of Japan.

Hidehiko Takara (M'97) received the B.S., M.E., and Ph.D. degrees in electrical engineering from the University of Keio, Tokyo, Kanagawa, Japan, in 1986, 1988, and 1997, respectively.

In 1988, he joined the NTT Transmission Systems Laboratories, Kanagawa, Japan. He is currently a Senior Research Engineer at the NTT Network Innovation Laboratories, NTT Corporation, Yokosuka, Kanagawa. His research interests include ultrahigh-speed/large capacity optical transmission systems and optical measurement techniques. He is a Member of the Institute of Electronics Information and Communication Engineers.

Akihide Sano (M'08) received the B.S. and M.S. degrees in physics and the $\mathrm{Ph} . \mathrm{D}$. degree in communication engineering from Kyoto University, Kyoto, Japan, in 1990, 1992, and 2007, respectively.

In 1992, he joined the NTT Transmission Systems Laboratories, Yokosuka, Kanagawa, Japan, where he was involved in research and development on highspeed optical communication systems. Since April 2005, he has been with the NTT Network Innovation Laboratories, Yokosuka. His current research interests include large-capacity long-haul fiber-optic communication systems. $\mathrm{He}$ is a Member of the IEEE Photonics Society and the Institute of Electronics, Information and Communication Engineers (IEICE) of Japan. He received the Best Paper Award of the First Optoelectronics and Communication Conference in 1996, the Young Engineer Award in 1999, and the Achievement Award from the IEICE in 2010.

Hiroto Kawakami (M'03) received the B.S. degree in physics from Kyoto Industrial University, Kyoto, Japan, in 1989, and the M.S. degree in physics from Hokkaido University, Sapporo, Japan, in 1991.

In 1991, he joined the NTT transmission Laboratories, Yokosuka, Japan, where he was involved in research and development of high-speed optical communications systems. His research interests include nonlinear effect in optical fiber and device control in transmission system. He is a Member of the OSA and the Institute of Electronics, Information and Communication Engineers of Japan. 
Yutaka Miyamoto (M'93) was born in Tokyo, Japan, on December 8, 1963. He received the B.E. and M.E. degrees in electrical engineering from Waseda University, Tokyo, in 1986 and 1988, respectively.

In 1988, he joined the NTT Transmission Systems Laboratories, Yokosuka, Japan, where he was involved in research and development on high-speed optical communications systems including the $10-\mathrm{Gb} / \mathrm{s}$ first terrestrial optical transmission system (FA-10G) using erbium-doped fiber amplifiers inline repeaters. He then joined the NTT Electronics Technology Corporation between 1995 and 1997, where he involved in the planning and product development of highspeed optical module at the data rate of $10 \mathrm{~Gb} / \mathrm{s}$ and beyond. Since 1997, he has been with the NTT Network Innovation Laboratories, Yokosuka, Japan, where he is involved in the research and development of optical transport technologies based on $40-$ and $100-\mathrm{Gb} / \mathrm{s}$ channel. He is currently the Senior Distinguished Researcher and Director at the Innovative Photonic Network Research Center, NTT Network Innovation Laboratories. His current research interest includes high-capacity optical transport systems with advanced modulation formats, digital signal processing, and space-division multiplexing. He is a Fellow of the IEICE.

Hirotaka Ono (M'96-SM'15) received the B.S., M.S., and Ph.D. degrees in applied physics from Tohoku University, Sendai, Japan, in 1993, 1995, and 2004, respectively. He joined the NTT Laboratories, Tsukuba, Ibaraki, Japan, in 1995. He was also a Visiting Research Fellow with the Optoelectronics Research Centre, University of Southampton, Southampton, U.K., from 2005 to 2006. He has been involved in research on optical fiber amplifiers, including Land S-band erbium-doped fiber amplifiers. He has also undertaken research on highly nonlinear fiber devices, photonic crystal fibers, and wavelength-divisionmultiplexing transmission systems. His current research interests include fiber and waveguide devices, including optical amplifiers, used for space-divisionmultiplexing systems. He is a Member of the Institute of Electronic, Information and Communication Engineers of Japan and the Japan Society of Applied Physics.

Manabu Oguma was born in Hokkaido, Japan, on April 7, 1966. He received the B.S. and M.S. degrees in applied physics from Tohoku University, Sendai, Japan, in 1989 and 1991, respectively.

He joined the Nippon Telegraph and Telephone Corporation, Atsugi, Japan, in 1991, where his research interest includes optical waveguide devices. $\mathrm{He}$ is a Member of the Institute of Electronics, Information and Communication Engineers, and the Japan Society of Applied Physics.

Yoshiteru Abe received the B.E. degree in electrical engineering, the M.E. degree in electronic device engineering, and the Dr. Eng degree in electrical engineering from Kyushu University, Fukuoka, Japan, in 1996, 1998, and 2005, respectively.

In 1998, he joined the Nippon Telegraph and Telephone Corporation Optoelectronics Laboratories, Tsukuba, Ibaraki, Japan. He is currently with the NTT Device Innovation Center, NTT Corporation, Atsugi, Kanagawa, Japan. His research interest includes optical fiber connectors.
Takashi Matsui was born in Hokkaido, Japan, on December 30, 1978. He received the B.E., M.E., and Ph.D. degrees in electronic engineering from Hokkaido University, Sapporo, Japan, in 2001, 2003, and 2008, respectively.

In 2003, he joined the NTT Access Network Service Systems Laboratories, Tsukuba, Ibaraki, Japan, where his research interests include optical fiber design and related maintenance techniques. He is a Member of the Institute of Electronics, Information and Communication Engineers of Japan.

Ryohei Fukumoto received the B.S. and M.S. degrees in applied physics from Hokkaido University, Sapporo, Japan, in 2011 and 2013, respectively.

He joined the Optics and Electronics Laboratory, Fujikura Ltd., Chiba, Japan, in 2013, and he is currently with the Advanced Technology Laboratory, Fujikura Ltd. He has been involved in research and development of multicore fibers and its fabrication technology.

Yoshimichi Amma received the B.S. and M.S. degrees in mechanical engineering from Yokohama National University, Yokohama, Kanagawa, Japan, in 2010 and 2012, respectively.

He joined the Optics and Electronics Laboratory, Fujikura Ltd., Chiba, Japan, in 2012. He is currently with the Advanced Technology Laboratory, Fujikura Ltd. He has been involved in research and development of multicore fibers and splicing technology for multicore fibers. He is a Member of the Institute of Electronics, Information and Communication Engineers.

Tsukasa Hosokawa received the B.S. and M.S. degrees from the Department of Metallurgy and Ceramics Science, Tokyo Institute of Technology, Tokyo, Japan, in 2009 and 2011, respectively. He joined the Optics and Electronics Laboratory, Fujikura Ltd., Chiba, Japan, in 2011. He is currently with the Advanced Technology Laboratory, Fujikura Ltd. He has been involved in research and development of rare-earth doped fibers. He is a Member of the Institute of Electronics, Information and Communication Engineers.

Shoichiro Matsuo (M'11) received the B.E. and M.E. degrees in electrical engineering from Kyushu University, Fukuoka, Japan, in 1988 and 1990, respectively, and the $\mathrm{Ph} . \mathrm{D}$. degree in production and information science from Utsunomiya University, Tochigi, Japan, in 2008.

He has been with Fujikura Ltd., Chiba, Japan, since 1990, where he has been working on research and development of transmission fibers for long-haul network and FTTH network and rare-earth-doped fibers, photonic bandgap fibers, and manufacturing technology of these optical fibers. He is currently the General Manager at the Advanced Technology Laboratory, Fujikura Ltd. He is an Author or Coauthor of more than 100 journals and conference papers and is an Inventor of more than 90 granted patents over the world. He is a Member of the Optical Society of America, the Japanese Society of Applied Physics, and the Institute of Electronics, Information and Communication Engineers. 
Kunimasa Saitoh (S'00-M'01) received the B.S., M.S., and Ph.D. degrees in electronic engineering from Hokkaido University, Sapporo, Japan, in 1997, 1999 , and 2001, respectively.

From 2001 to 2005, he was a Research Associate with the Graduate School of Engineering, Hokkaido University. From 2005 to 2013, he was an Associate Professor with the Graduate School of Information Science and Technology, Hokkaido University, where he became a Professor in 2013. He is the Author of more than 160 research papers in refereed international journals and 190 refereed conference presentations. His research interests include fiber optics, nanophotonics, integrated optical devices, and computer-aided design and modeling of guided-wave devices. From 1999 to 2001, he was a Research Fellow of the Japan Society for the Promotion of Science. He is a Member of the Optical Society of America, and the Institute of Electronics, Information and Communication Engineers (IEICE). He received the Excellent Paper Award and the Young Scientist Award from the IEICE in 1999 and 2002, respectively, and the Young Scientists Prize of the Commendation for Science and Technology from the Ministry of Education, Culture, Sports, Science, and Technology, Government of Japan, in 2008. From 2009 to 2010, he served as a Secretary/Treasurer of the IEEE Sapporo Section.

Makoto Yamada (SM'14) received the B.S., M.E., and Ph.D. degrees in electrical engineering from the Technical University of Nagaoka, Niigata, Japan, in 1983, 1985, and 1998, respectively.

In 1985, he joined the NTT Laboratories, where he was involved in research on guided-wave optical devices. Since 1989, he has been involved in research on optical fiber amplifiers. In 2008, he joined Osaka Prefecture University, Sakai, Japan, where since 2013, has been a Professor. His research interests include design and control for optical amplifiers and other components in optical networks. He is a Senior Member of the Institute of Electronics, Information and Communication Engineers.
Toshio Morioka (M'92) was born in Hyogo, Japan. He received the B.E. degree in applied physics from Waseda University, Tokyo, Japan, in 1982, the M.S. degree in optical sciences from the University of Arizona, Tucson, AZ USA, in 1984, and the M.S. and Ph.D. degrees in physics and applied physics from Waseda University in 1985 and 1995, respectively.

In 1985, he joined the Yokosuka Electrical Communication Laboratory, Nippon Telegraph and Telephone (NTT) Corporation. From 1996 to 1999, he was with the NTT Bureau de Gen 'eve, Geneva, Switzerland, and from 2005 to 2008, he was with the National Institute of Information and Communications Technology, Japan. In 2011, he joined the High-Speed Optical Communications Group, DTU Fotonik, Kgs. Lyngby, Denmark, where since 2013, has been a Full Professor. He has been involved in pioneering works on all-optical signa processing technologies for next-generation optical communication systems; he proposed the use of the optical Kerr effect for all-optical demultiplexing in 1986, the use of supercontinuum generation for OTDM/WDM sources in 1993, and demonstrated 1-Tb/s OTDM/WDM $(100 \mathrm{~Gb} / \mathrm{s} \times 10$ channels $)$ transmission in 1996. In 2008, he organized extremely advanced transmission initiative in Japan, to initiate space-division multiplexing research based on novel fibers (multicore/few mode fibers) aiming at well over $\mathrm{Pb} / \mathrm{s}$ transmission, which led to the first one Pbit/s transmission experiment in 2012. He holds 59 issued patents.

Dr. Morioka received the Sakurai Memorial Award from the Optoelectronic Industry and Technology Development Association, Japan, in 1996; the Achievement Award and Kobayashi Memorial Award from the Institute of Electronics, Information and Communication Engineers of Japan (IEICE) in 1995; the IEE Electronics Letters Premium Award in 1997; and the Paper Award from the Laser Society of Japan in 2003. He is a Fellow of the OSA and the IEICE and a Member of the IEEE Photonics Society/Communications Society. He was a Program and General Cochair of the OSA topical meeting on the Nonlinear Guided Waves and Their Applications in 1995 and 1996, respectively. 